\title{
EHMTI-0332. Health care utilisation for primary headache disorders: insights from Karnataka, India
}

\author{
$\mathrm{G} \mathrm{Rao}^{1 *}, \mathrm{G}$ Gururaj ${ }^{1}, \mathrm{G}$ Kulkarni $^{2}$, DK Subbukrishna ${ }^{3}$, T Steiner $^{4}$, L Stovner $^{5}$ \\ From 4th European Headache and Migraine Trust International Congress: EHMTIC 2014 \\ Copenhagen, Denmark. 18-21 September 2014
}

\section{Background}

Globally, primary headache disorders are neglected and inadequately treated. There is little information on management or health-care utilisation for headache disorders from the Indian sub-continent.

\section{Aim}

To fill this knowledge gap in Karnataka State, south India.

\section{Methods}

In a population-based survey, 2,329 randomly selected, biologically unrelated adults (18-65 years) (1,141 male, 1,188 female; 1,226 urban, 1,103 rural) were interviewed using a validated structured questionnaire. Ethics approval and informed consent from participants were obtained.

\section{Results}

Headache was reported by 1,488 persons (crude annual 1 -year prevalence $63.9 \%$ ) with a mean age of $37 \pm 12$ years, $58 \%$ females and $53 \%$ rural-dwelling. Only $24.7 \%$ (32.4\% rural, $16.2 \%$ urban) had sought medical help. Of these, $80.6 \%$ had seen a primary-care doctor and $15.8 \%$ a specialist. Greater proportions of urban dwellers (38.6\%) and females $(17.7 \%)$ had consulted specialists. Consultation rates were higher for migraine (41.9\% overall) but, even among those with high disability assessed as lost productive time by HALT questionnaire, did not exceed $50 \%$ (HALT grade 1: 27.3\%; grade 2: $39.0 \%$; grade 3: $45.7 \%$; grade $4: 45.5 \%)$. Consultation rates were much higher for any headache occurring on $\geq 15$ days/month $(72.5 \%)$ and medication-overuse headache (78.6\%).

\section{Conclusion}

Despite the high prevalence of primary headache disorders, health-care utilisation is poor. The primary care physician is consulted most often, which is where headache services should be built. Structured headache services require primary-care physicians trained in managing headache disorders, facilitated links to secondary care when needed, but also improved awareness among people with headache so that they use them.

No conflict of interest.

\section{Authors' details}

${ }^{1}$ Epidemiology Centre for Public Health, National Institute of Mental Health and Neurosciences, Bangalore, India. ${ }^{2}$ Neurology, National Institute of Mental Health and Neurosciences, Bangalore, India. ${ }^{3}$ Bio-statistics, National Institute of Mental Health and Neurosciences, Bangalore, India. ${ }^{4}$ Neuroscience, Imperial College, London, UK. ${ }^{5}$ Neuroscience, Norwegian University of Science and Technology, Trondheim, Norway.

Published: 18 September 2014

doi:10.1186/1129-2377-15-S1-D48

Cite this article as: Rao et al:: EHMTI-0332. Health care utilisation for primary headache disorders: insights from Karnataka, India. The Journal of Headache and Pain 2014 15(Suppl 1):D48. 